\title{
Recent guidelines in preoperative assessment, premedication\&perioperative documentation
}

\section{Sara M.Galal,Abdelhady A.Helmy,Ahmed M. Ahmed Abdelmaabood, El Hadad A.Mousa}

Department of Anesthesia \& ICU, Faculty of Medicine, Sohag University

\begin{abstract}
Introduction: The corner stone of an effective preoperative evaluation are the history and physical examination, which should include a complete account of all medications taken by the patient in the recent past, and responses and reactions to previous anesthetics.Premedication is the administration of medication before anesthesia. Premedication is used to prepare the patient for anesthesia and to help provide optimal conditions for surgery. The anesthetic record is usually the sole documentation of an anesthetist's interaction with his or her patient.

Aim of the work: This work aims to review the recent guidelines in preoperative assessment, premedication and perioperative documentation to reach a conclusion and a protocol of management that can be applicable in our hospital.

Materials and methods: This is a clinical review of recent guidelines in preoperative assessment, premedication, perioperative documentation based on explore other protocols and researches.

The strategy followed in doing this review is as follow; Exploring the data allowed for the recent guidelines in preoperative assessment, premedication, perioperative documentation

1-Sites to be visited.

2-Recent searches in the last 5 years.

3-Search terms including preoperative assessment, patient preparation ,premedication ,perioperative documentation, anesthetic record .

4-Bibliographies of articles are also searched for relevant articles.

5-English language studies and full text studies will be preferred.

Conclusion: The goals of the preanesthesia evaluation are twofold: first, to ensure that patients can safely tolerate anesthesia for planned surgical procedures; and second, to mitigate risks associated with the overall perioperative period, such as postoperative pulmonary or cardiac complications. The patient's comorbidities and planned procedure must be considered when managing medications in the perioperative period.

Accurate and thorough documentation is an essential element of high quality and safe medical care, and accordingly a basic responsibility of physician anesthesiologists.
\end{abstract}

Keywords: Preoperative assessment, Premedication, Anesthetic record

\section{Introduction}

Preoperative Assessment :The corner stones of an effective preoperative evaluation are the history and physical examination, which should include a complete account of all medications taken by the patient in the recent past, and responses and reactions to previous anesthetics1.The history and physical examination, rather than routine laboratory, cardiovascular, and pulmonary testing, are the most important components of the preoperative evaluation2.The preoperative evaluation guides the 
anesthetic plan, inadequate preoperative planning and incomplete patient preparation are commonly associated with anesthetic complications $\mathbf{1}$.

Premedicationis the administration of medication before anesthesia. Premedication is used to prepare the patient for anesthesia and to help provide optimal conditions for surgery. This includes: Reduction of anxiety and pain, promotion of amnesia, reduction of secretions, reduction of volume and $\mathrm{pH}$ of gastric contents (to avoid Mendelson's syndrome),reduction of postoperative nausea and vomiting, enhancing the hypnotic effects of general anesthesia, reduction of vagal reflexes to intubation, Specific indications - e.g., prevention of infective endocarditis 3 .

Documentation:The anesthetic record is usually the sole documentation of an anesthetist's interaction with his or her patient. The record generally serves as a concise document of the relevant preoperative assessment, intraoperative anesthetic administration and physiological data, as well as the anesthetist's orders for the immediate postoperative management. The lack of clear and adequate documentation has not only medico-legal implications, but can potentially affect the quality and safety of patient care. Examples include the documentation of an unexpected difficult airway event or an allergic reaction to an administered medication. It is not surprising, therefore, that the importance of maintaining an adequate, accurate, and legible anesthetic record has been emphasized by numerous professional bodies including The Australian and New Zealand College of Anesthetists (ANZCA2009)The American Society of Anesthesiologists (ASA 2013)and The Canadian Anesthesiologists Society (CAS)4.

Aim of the work
This work aims to review the recent guidelines in preoperative assessment, premedication and perioperative documentation to reach a conclusion and a protocol of management that can be applicable in our hospital.

\section{Review}

Patients undergoing surgery today are older, have more chronic medical conditions, and are taking more medications than patients a decade ago5. Complications are frequent, and cardiovascular events remain the leading cause of perioperative death6. The annual cost of perioperative cardiovascular morbidity in the United States alone is more than $\$ 20$ billion 7 . The preoperative evaluation holds the potential to reduce complications and health care costs. Yet a standard preoperative evaluation has not been defined, and routine laboratory, cardiovascular, and pulmonary tests are often performed without justification8. The Joint Commission for the Accreditation of Hospitals requires all surgical patients to have a history and physical examination documented in the medical record within 30 days before surgery, but it does not define the components of the history or physical examination. Preoperative assessment and preparation is a process involves primary care, anesthesia and other specialties8. The goals of the preanesthesia evaluation are twofold: first, to ensure that patients can safely tolerate anesthesia for planned surgical procedures; and second, to mitigate risks associated with the overall perioperative period, such as postoperative pulmonary or cardiac complications9.In pursuit of these goals, the preanesthesia evaluation offers opportunities to perform focused clinical examinations, better document comorbid illness, reduce the patient's (and family's) anxiety through education, optimize preexisting medical conditions, make 
selective referrals to medical specialists (e.g., pulmonologists, cardiologists), order specialized preoperative investigations (e.g., cardiac stress tests), initiate interventions intended to decrease perioperative risk (e.g., $\beta$-adrenergic blockers), discuss aspects of perioperative care (e.g., anticipated risks, fasting guidelines, regional anesthesia), and arrange appropriate levels of postoperative care (e.g., admission to a critical care unit)9. The basics of the preoperative evaluation are a comprehensive history, a detailed physical examination, and selected laboratory tests10.A complete review of systems to look for undiagnosed disease or inadequately controlled chronic disease. The review of systems, in conjunction with the medical history, can also identify risk factors for perioperative complications, such as alcohol or tobacco use, recent chest pain, history of deep venous thrombosis, or prior hospitalization for asthma11. The physical examination should build on the information gathered during the history. For example, patients with identified chronic organ diseases such as congestive heart failure or chronic obstructive lung disease should be evaluated for uncompensated disease. Patients with a history of heavy alcohol use should be assessed for stigmata of chronic liver disease with concomitant concern for postoperative alcohol withdrawal syndromes and delirium11.In the preoperative assessment of the cardiovascular system, much research has been built on the foundations of the 1977 study by Goldman et al. which created the "Goldman Criteria". Within this study it was found that preoperative identifiable risks such as a third heart sound, jugular venous distention, myocardial infarction within six months, >5 premature ventricular contractions, non-sinus rhythm or presence of premature atrial contractions on preoperative electrocardiogram increased the likelihood of perioperative cardiac complications (PCC's)12. Also included with these risk factors were age greater than 70, intraperitoneal, intrathoracic, aortic or emergency surgery, valvular aortic stenosis and poor general medical health. In 1999 , Lee et al. modified and improved the predictability of the Goldman criteria creating the Revised Cardiac Risk Index (RCRI) Score. The RCRI created six independent variables of risk stratification. These variables were high risk surgery, history of ischemic heart disease, history of congestive heart failure, cerebral vascular disease, insulin controlled diabetes and serum creatinine greater than 2.0 $\mathrm{mg} / \mathrm{dL14}$. Today, current guidelines for preoperative cardiovascular risk stratification have been built on the Goldman and Lee studies and validated by the American College of Cardiology and American Heart Association's (ACC/AHA) 2002 and later revised 2007 Guidelines on Perioperative Cardiovascular Evaluation and Care for Non-cardiac Surgery13. Because preoperative cardiovascular assessment has been at the forefront of clinical inquiry to a patient's overall risk of surgery, it seems that renal assessment has often been overlooked. Acute kidney injury (AKI) in the postoperative period has been defined by the Acute Kidney Injury Network (AKIN) consensus group as an abrupt (within 48 hours of surgery) reduction of kidney function seen as a 1.5 times increase in serum creatinine from baseline or less than $0.5 \mathrm{ml} / \mathrm{kg}$ of urine output in 6 hours 14 . Current research with regards to preoperative risk factors for $\mathrm{AKI}$ is largely procedure focused with much attention on cardiovascular surgery. 
Smaller numbers of studies have shownindependent risk factors for AKI within the general surgery population. In a retrospective study (of noncardiovascular patients) found 9 risk factors for AKI in general surgery 15. These risk factors were, age greater than 56, male gender, active congestive heart failure, ascites, hypertension, emergency surgery, intraperitoneal surgery, mild to moderate renal insufficiency, and medication controlled diabetes. These risk factors were accompanied in the study with a 5 level risk stratification tool that interpreted \% incidence of AKI and hazard ratios based on the number of risk factors a patient had 15.Postoperative pulmonary complications contribute significantly to overall perioperative morbidity and mortality rates. Such complications account for about $25 \%$ of deaths occurring within 6 days of surgery. The frequency rate of these complications varies from 5-70\%. This wide range is due to variations among studies in the definition of postoperative pulmonary complications, as well as variability in patient- and procedure-related factors 16. The goal of perioperative pulmonary management is to identify patients at high risk of significant postoperative pulmonary complications, so that appropriate interventions can be provided to minimize that risk16. With the help of the RIFLE and then AKIN studies, definitions and stratification of AKI were made, however, the research guiding current preoperative assessments in certain surgical patients is still developing.
Increasing the evidence base of this subject will help guide future research in risk modification for patients at risk for AKI. Patient frailty is commonly used as an indication of patient aging and or the loss of physiologic reserve 15. Patients with liver disease who undergo surgery have an increased risk of morbidity and mortality 17. The optimal management of such patients requires the following:

a. Diagnosis of the underlying liver disease.

b. Assessment and stratification of the risk of surgery.

c. Estimation of functional hepatic reserve.

d. Correction of underlying conditions if feasible.

e. Hepatic hemodynamic evaluation and identification of the site of upper gastrointestinal hemorrhage, if present Impairment of the liver functions increases the risks of surgery and anesthesia in several ways, including the following 18:

1. Bleeding risk may increase because of coagulopathy.

2. Susceptibility to infection is increased due to altered functions of the hepatic reticuloendothelial cells and changes in the immune system and portal hypertension.

3. Reduced hepatic blood flow.

4. Altered drug metabolism18.

The most commonly used method by anesthesiologists to assess overall perioperative risk is the ASA physical status (ASA-PS) classification system (Table 2) . 
Category* Definition

ASA-PS 1

ASA-PS 2

ASA-PS 3

ASA-PS 4

ASA-PS 5

ASA-PS 6
A normal, healthy patient.

A patient with mild systemic disease.

A patient with severe systemic disease.

A patient with severe systemic disease that is a constant threat to life.

A moribund patient who is not expected to survive without the operation.

A declared brain-dead patient whose organs are being removed for donor purposes.

$\boldsymbol{A S A - P S}$, American Society of Anesthesiologists physical status.

*The addition of "E" to the classification category indicates emergency

\section{Table 2. American sociaty of Anesthethiologists Physical ststus classification.}

This classification system, which was developed in 1941 by Meyer Sakland at the request of the ASA, 19 was originally intended to facilitate collection and comparison of statistical data in anesthesia. The ASA-PS classification system seeks to describe a patient's preoperative medical status, but it does not consider risks inherent to the planned surgical procedure. Although not intended to guide estimation of patients' risks for anesthesia and surgery, the ASA-PS is often used for this very purpose, especially given its simplicity of use. Indeed, several studies have shown a correlation of ASA-PS scores with postoperative mortality and majorcomplications 19. Classification schemes have been proposed for assessing operative risk, such as the Johns Hopkins risk classification system (Table 3) and the strata proposed by the ACCF/AHA

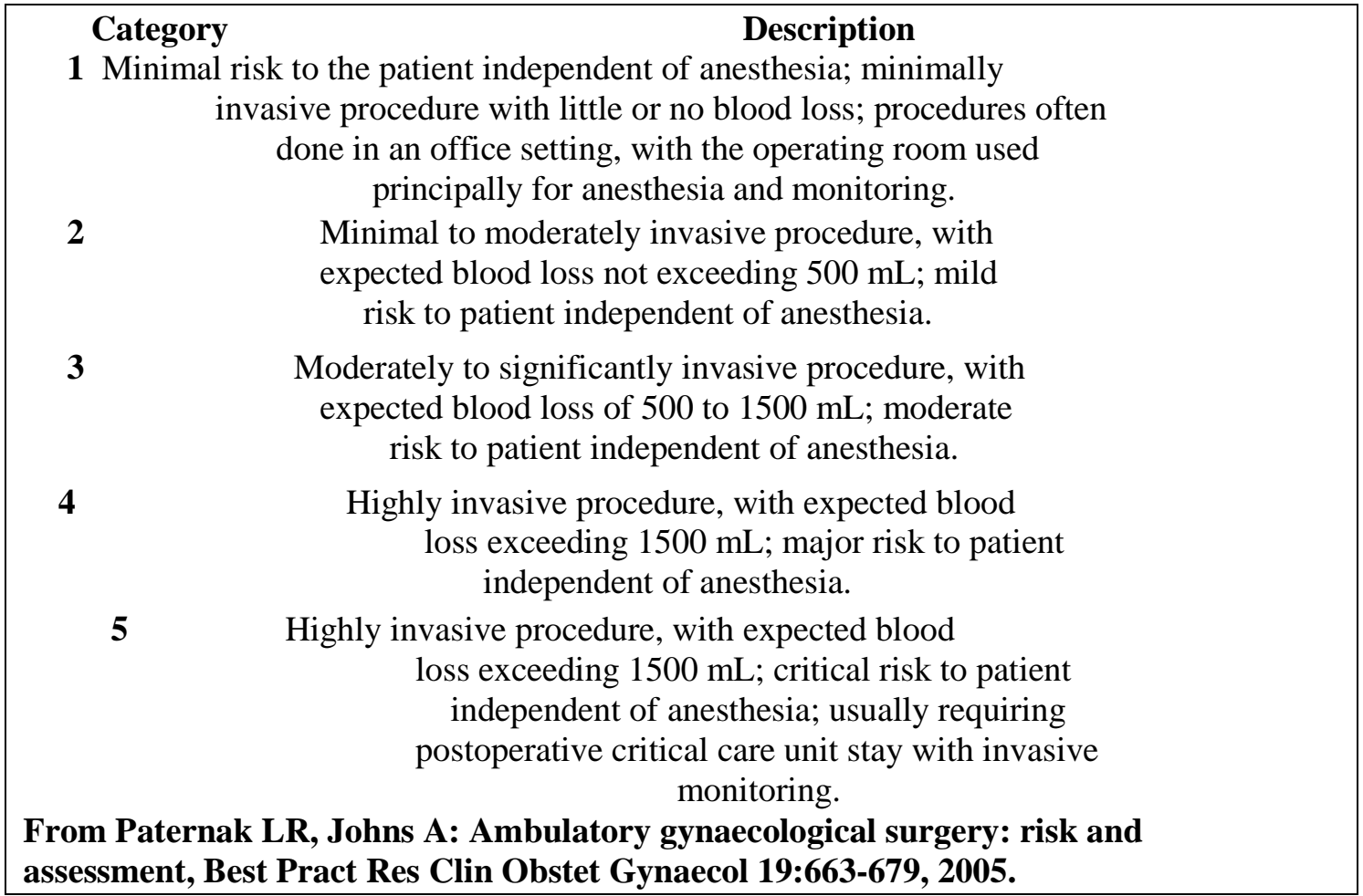

Table 3. Johns Hopkins surgery Risk classification system.9

For noncardiac surgery, the American College of Surgeons National Surgical Quality Improvement Project (NSQIP) risk calculator is available on the Internet and provides 
an estimate of risk based on patients' comorbidities and proposed surgical procedures. Large multinational prospective epidemiologic studies of surgical patients, which included accurate capture of perioperative characteristics and outcomes, 20will likely help lead to the development of similar indices for noncardiac surgery. Initial results from the VISION study, a multinational prospective cohort study of 15,133 individuals who were 45 years old or older and who underwent noncardiac surgical procedures, identified several independent predictors of postoperative 30-day mortality, including age, operative procedure, nonelective surgery, and specific comorbid conditions (Table 4) $\mathbf{. 1 9 0}$

\section{NCARDISURGERY}

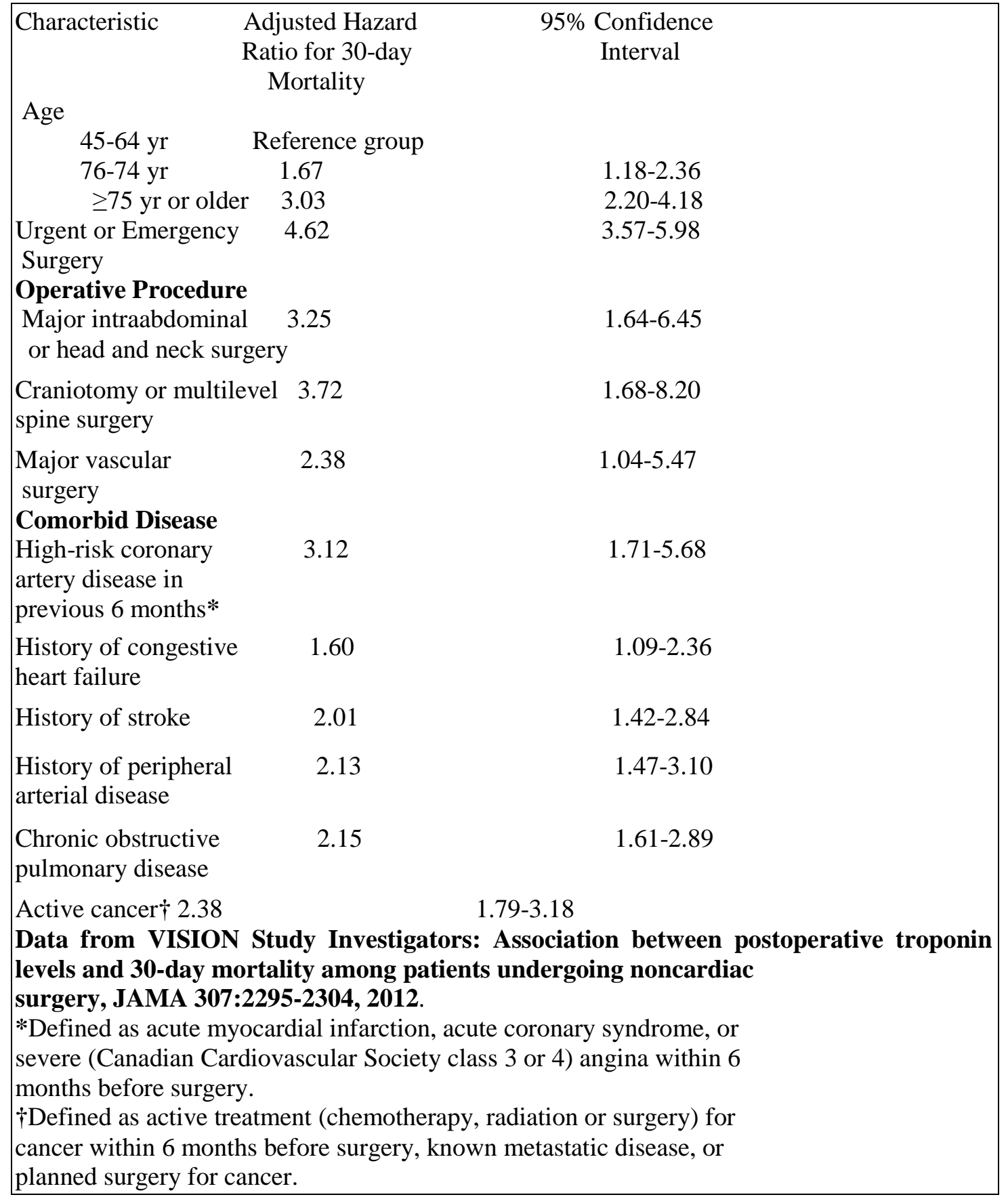

YTable 4. Preoperative Predicators of 30 Day Mortality after non cardiac surgery.19 
The patient's comorbidities and planned procedure must be considered when managing medications in the perioperative period. Some medications have beneficial effects during surgical procedures, whereas others may be detrimental. In some cases, withdrawal of medications suddenly can have a negative effect $\mathbf{2 0}$. Outlines the medications to continue or discontinue

preoperatively.Antihypertensive

medications should generally be continued preoperatively. Stopping $\boldsymbol{\beta}$ blockers or $\boldsymbol{\alpha 2}$ - agonists (e.g., clonidine) may be associated with rebound hypertension. For patients who cannot tolerate hypotension, consideration may be given to discontinuing ACEI or ARB agents 12 to 24 hours preoperatively. Continuation of these agents up to the morning of surgery can lead to increased risks for intraoperative hypotension, 20especially in individuals receiving concomitant diuretic therapy 21. This hypotension can be refractory to fluid administration and typical vasopressors, although vasopressin has been used successfully to treat hypotension in these instances. The benefit of withholding ACEI and ARB agents may be especially pertinent for cardiac surgery, complex spine surgery, procedures involving significant blood loss or fluid shifts, patients taking multiple antihypertensive medications, or procedures involving intraoperative positioning that may impair hemodynamics22.Patients with either type 1 or type 2 diabetes should discontinue short-acting insulin while fasting. The exception to this rule pertains to diabetic patients with continuous subcutaneous insulin infusion pumps. Such individuals should instead continue their infusion at the lowest basal rate, which is usually the nighttime fasting rate23.On the day of surgery, patients with type 1 diabetes typically need to take a small amount (one third to one half) of their usual morning dose of intermediateacting or long-acting insulin (e.g., lente, NPH) to avoid diabetic ketoacidosis. Patients with type 2 diabetes can either take no insulin or up to one half of their usual dose of intermediate- acting, long-acting, or combination (e.g., 70/30 preparations) insulin on the morning of surgery 23. These agents are generally continued up until the time of the surgical procedure because of their beneficial effects and the potential for negative consequences if discontinued.

NSAIDs have reversible antiplatelet effects; hence, once the drugs have been eliminated, platelet function returns to normal. Concomitant NSAID use does not appear to increase the risk of spinal hematoma with neuraxial anesthesia23. Patients taking steroids on a long-term basis should take their usual dose on the day of surgery. Stress-associated adrenal insufficiency may occur in patients taking steroids unless additional steroid therapy is instituted perioperatively. Approximately 5 to $7.5 \mathrm{mg}$ of prednisone is equivalent to the normal daily adrenal output of cortisol, which is approximately 30 $\mathrm{mg}$. The HPA axis is not suppressed in patients who take less than $5 \mathrm{mg} /$ day of prednisone or its equivalent24. In patients who take 5 to $20 \mathrm{mg} /$ day of prednisone or its equivalent for 3 or more weeks, the HPA axis may or may not be suppressed. Patients who take more than $20 \mathrm{mg} /$ day of prednisone or its equivalent for 3 or more weeks have suppression of their HPA axis. The risk of adrenal insufficiency remains for up to 1 year after the cessation of highdose steroid therapy 21. Postmenopausal hormone replacement therapies that contain 
estrogen increase the risk of thromboembolic events 24. It may therefore be reasonable to discontinue these medications before operations. Estrogens must be stopped approximately 1 month preoperatively for coagulation function to return to baseline. Most modern oral contraceptives contain low doses of estrogen that minimally increase thromboembolic risk21. This long withdrawal period specifically applied to MAOIs that caused irreversible inhibition of MAO. Some newer agents, such as moclobemide, cause reversible enzyme inhibition and have effects lasting less than 24 hours. Preoperative withdrawal of these drugs has potential risks. Specifically, case reports of suicides or severe depression following discontinuation of MAOIs have been reported in the literature 24 . Thus, the safest approach may be to continue these drugs and adjust the anesthetic plan to make changes such as avoiding meperidine and indirectacting vasopressors (e.g., ephedrine). Details regarding a patient's MAOI use must be clearly communicated to health care providers on the day of the surgical procedure. Patients receiving tricyclic antidepressants require a preoperative ECG, given the potential for associated prolongation of the QT interval23. Patients taking lithium require evaluation of electrolytes, BUN, and creatinine. Discontinuation of lithium has also been associated with suicide. Similarly, abrupt discontinuation of SSRIs can also cause adverse effects, including dizziness, headache, nausea, irritability, visual disturbances, and sensations of electric shocks. Complementary and alternative medications mayinteract with anesthetic agents, alter effects of prescriptionmedications, and increase bleeding. In addition, many patients do not consider these agents "medications" and hence do not include them in a list of theirmedications unless specifically asked23. Accurate and thorough documentation is an essential element of high quality and safe medical care, and accordingly a basic responsibility of physician anesthesiologists. Anesthesia care is a continuum including three general phases of care: preanesthesia, intraoperative/intraprocedural

anesthesia and postanesthesia care. To contribute to accuracy in medical records and to facilitate any future necessary chart review, anesthesiologists should ensure that accurate and thorough documentation is accomplished in all three phases of anesthesia relatedcare.25A. Patient interview to assess:

1. Patient and procedure identification

2. Anticipated disposition .

3. Medical history - includes patient's ability to give informed consent.

4. Surgical History (PSHx) .

5. Anesthetic history .

6. Current Medication List (preadmission and postadmission)

7. Allergies/Adverse Drug Reaction (including reaction type)

8. NPO status.

9. Documenting the presence of and the perioperative plan for existing advance directives.

B. Appropriate physical examination, including vital signs, height and weight and documentation of airway assessment and cardiopulmonary exam.

C. Review of objective diagnostic data (e.g., laboratory, ECG, X-ray) and medical records.

D. Medical consultations when applicable.

E. Assignment of ASA physical status, including emergent status when applicable.

F. The anesthetic plan - including plans for post-anesthesia care and pain management. 
G. Documentation of informed consent (to include risks, benefits and alternatives) of the anesthetic plan and postoperative pain management plan.

H. Appropriate premedication and prophylactic antibiotic administrations (if indicated) 25

Documentation and communication of information on pre-operative preparation are essential. Electronic systems should be considered to enable the capture and sharing of information, support risk identification and allow data to be collected and available for audit and research purposes 26. Preoperative assessment should take place as early as possible in the patient's care pathway so that all essential resources and obstacles can be anticipated before the day of the operation, including discharge arrangements27. As a result of the assessment, the appropriate level of post-operative care can be determined and booked in a daysurgery facility, ward, highdependency unit (level 2 care), or critical care unit (level 3 care), enabling both optimum care and efficient planning 28Patients should be admitted to a ward or alternative facility with sufficient time before the operating list on which they are scheduled. If an adequate pre-operative assessment has been performed, admission can be on the day of surgery but it remains essential that the anesthetist who will be administering the anesthetic is able to confirm the findings of the assessment and agree final details with the patient. Discharge planning should be started as soon as the patient opts for surgery so that all essential resources and obstacles to discharge can be identified and dealt with, including liaison with social services26.This will minimize late cancellation of operations and reduce the length of stay in hospital28. This list should be rewritten or reprinted, including a date and time of the update. Written guidelines should cover the policy for the collection of patients from the ward or admissions unit, as well as the handover by ward staff to a designated member of the operating department staff. Eighty per cent of patients undergoing elective surgery can expect to follow a daysurgery pathway. If in-patient care is necessary, an enhanced recovery pathway is now considered to provide optimum care and the preoperative service should ensure that patients are clear about their own responsibilities and expected length of stay 29. There should be provision for carbohydrate drinks to take pre-operatively where appropriate. A designated pharmacist should be available to provide advice and input into anesthetic and preoperative assessment. This level of input may range from ad-hoc advice through to designated pre-operative assessment pharmacists, preferably with prescribing rights, who can undertake medicines reconciliation, produce perioperative medication plans and provide specialist advice29. The competent patient has a fundamental right, under common law, to give, or to withhold, consent to examination, investigation and treatment . No other person can consent to treatment on behalf of any adult. There should be a local process and policy in place for patients who lack capacity that conforms to national guidance and the Mental Capacity Act 28. The scope of the authority that has been given by a patient should not be exceeded except in an emergency. In an emergency clinical situation, where it is not possible to find out a patient's wishes, a patient must be treated without their consent, provided the treatment is immediately necessary to save their life or to prevent a serious deterioration of their condition. The treatment provided should be the least restrictive of the patient's future 
choices28. The patient must be made aware of alternative treatment options, or the option for no treatment at all. It is acceptable to recommend one of the alternatives but, as the GMC states: 'The doctor may recommend aparticular option which they believe to be best for the patient, but may not put any pressure onto the patient toaccept their advice. 30. Where risks of adverse patient outcome with surgery are identified as being high, the pre-operative assessmentconsultation facilitates shared patient discussion, which may result in a well-informed individual opting fornon-surgical management. Under such circumstances the decision-making process should be endorsed throughclose collaborative discussion with surgical colleagues (ideally a pre-operative MDT meeting)31. The variability in the words that patients and physicians use to describe symptoms is a common problem. The use of lay language and the recording of symptoms in ordinary words lead to greater interobserver agreement among practitioners and can prevent communication errors that are common in medical care. Common errors occur when diagnostic labels such as "angina" are written in the medical record when the patient actually complained of "chest pain." Conversely, patients rarely describe true angina or myocardial infarction (MI) as chest pain 10. The legal requirements and guidelines for anesthesia record design are quite general. For example, the portio of the regulations under the Public Hospitals Act of the Canadian Province of Ontario 9 which apply to anesthesia records states: Where an anesthetic referred to in subsection or is administered to a patient, the anesthetist who administers the anesthetic shall prepare ananesthetic report with respect to the patient that shows the following:(a) The medications given to the patient in contemplation of anesthesia.(b)The patient airway, circuit and monitors used on the patient.(c) The anesthetic agents used, the methods of administration of the agents and the proportions or concentrations of all agents administered by inhalation to the patient.(d) The names, quantities and times of all drugs given by injection to the patient.(c) The duration of the anesthesia on the patient.(f) The estimated fluid loss of the patient.(g) The quantities and type of all blood products and other fluids administered intravenously to the patient during the operation. (h) The vital signs of the patient before, during and after anaesthesia.32

The review recommendationsfordocumentation ofIntraoperative/procedural anesthesia care from Committee on Quality Management and Departmental Administration (QMDA)

Approved by The American Society of Anesthesiologists are as follows:

A. Immediately prior to the start of anesthesia care and anesthesia procedures:

1. Patient re-evaluation

2. Confirmation of availability of and appropriate function of all necessary equipment, medications and staff.

B. Physiologic monitoring data** (e.g., recording of results from routine and nonroutine monitoring devices).

C. Medications administered: dose, time, route, response (where appropriate).

D. Intravenous fluids: type, volume and time.

E. Technique(s) used.

F. Patient positioning and actions to reduce the chance of adverse patient effects/complications related to positioning. 
G. Additional Procedures performed: vessel location, catheter type/size, specific insertion technique (e.g., sterile technique, use of ultrasound), actions to reduce the chance of related complications (ex., catheter based infection prevention measures), stabilization technique and dressing. 25

\section{Postanesthesia documentation}

A. Patient status at transfer of care to staff in a Postanesthesia Care Unit (PACU) or an area which provides equivalent postanesthesia care (e.g. ICU, SDS or floor nurse .(

B.If the PACU is bypassed, criteria demonstrating that patient status at transfer of care are appropriate .

C.It is not the responsibility of the anesthesiologist to document the patient's condition throughout the PACU stay or when leaving the PACU .

D.Significant or unexpected postprocedural events/complications .

E.Postanesthesia evaluation documenting physiologic condition and presence/absence of anesthesia related complications or complaints. .QMDA approved by ASA 201525

\section{Conclusion}

\section{Preoperative assessment}

The preoperative evaluation holds the potential to reduce complications and health care costs. The preoperative evaluation can help influence and thereby improve perioperative care . The goals of the preanesthesia evaluation are twofold: first, to ensure that patients can safely tolerate anesthesia for planned surgical procedures; and second, to mitigate risks associated with the overall perioperative period, such as postoperative pulmonary or cardiac complications. When the patient is at very high risk for adverse perioperative outcomes, the anesthesiologist may recommend an alternative nonoperative or less invasive treatment. Diagnostic tests, such as chest radiographs (x-ray studies) and ECGs, helped with only $3 \%$ of diagnoses, whereas special tests (e.g., cardiac stress tests) assisted with $6 \%$. In respiratory, urinary, and neurologic conditions, history taking is the most important diagnostic method. The basics of the preoperative evaluation are a comprehensive history, a detailed physical examination, and selected laboratory tests. The history is the most important component of the preoperative evaluation.A complete review of systems to look for undiagnosed disease or inadequately controlled chronic disease.An extensive medication history. This should include over-the-counter medications and herbal supplements. Recent use of anticoagulants, aspirin, and nonselective nonsteroidal antiinflammatory drugs (NSAIDs) must be specifically sought. Allergies, surgical and anesthetic history, functional status. The physical examination should build on the information gathered during the history. Anything found in the review of systems; CVS,renal \&pulmonary systems, liver and other systems should be addressed . Unexpected abnormal findings on the physical examination should be fully characterized and investigated before elective surgery.Other Important Preoperative Investigations such as pregnancy testing, general preoperative risk assessment like ASA classification and Johns Hopkins risk classification system should be performed.

\section{Preoperative Medication}

The patient's comorbidities and planned procedure must be considered when managing medications in the perioperative period. Some medications have beneficial effects during surgical procedures, whereas others may be detrimental. In some cases, withdrawal of medications suddenly can have a negative 
effect.For example antihypertensive Medications, outlines the medications to continue or discontinue preoperatively.Antihypertensive medications should generally be continued preoperatively. Stopping $\beta$ blockers or $\alpha 2$ - agonists (e.g., clonidine) may be associated with rebound hypertension. For patients who cannot tolerate hypotension, consideration may be given to discontinuing ACEI or ARB agents 12 to 24 hours preoperatively. Continuation of these agents up to the morning of surgery can lead to increased risks for intraoperative hypotension, especially in individuals receiving concomitant diuretic therapy. Diuretics are generally discontinued preoperatively, with the exception of thiazide diuretics (e.g., hydrochlorothiazide, chlorthalidone) if taken for hypertension .Medications for diabetic patients .Patients with either type 1 or type 2 diabetes should discontinue short-acting insulin while fasting. The exception to this rule pertains to diabetic patients with continuous subcutaneous insulin infusion pumps. Such individuals should instead continue their infusion at the lowest basal rate, which is usually the nighttime fasting .Metformin does not have to be routinely discontinued before the day of surgery. Sulfonylurea agents with very long halflives (e.g., chlorpropamide) can cause hypoglycemia in the fasting patient and should therefore be withheld on the day of surgery. Non steroidal antiinflammatory medications :are generally continued up until the time of the surgical procedure because of their beneficial effects and the potential for negative consequences if discontinued. .Patients taking steroids on a long-term basis should take their usual dose on the day of surgery. Stress-associated adrenal insufficiency may occur in patients taking steroids unless additional steroid therapy is instituted perioperatively. The stress associated with surgery, trauma, or infection leads an intact HPA to increase adrenal output of glucocorticoids. In most patients, the circulating cortisol concentration then returns to normal within 24 to 48 hours .Postmenopausal hormone replacement therapies that contain estrogen increase the risk of thromboembolic events .Other drugs like antidepressants, antipsychotics, and benzodiazepines are best maintained to avoid exacerbations of symptoms. Anxiolytics are continued up until the time of the procedure. Patients taking lithium require evaluation of electrolytes, BUN, and creatinine. .

\section{Perioperative Documentation}

Accurate and thorough documentation is an essential element of high quality and safe medical care, and accordingly a basic responsibility of physician anesthesiologists. .

Anesthesia care is a continuum including three general phases of care. Preanesthesia, intraoperative/intraprocedural anesthesia and postanesthesia care.

\section{Preoperative documentation} including documentation of preanesthesia Evaluation of history, vital signs, and cardiopulmonary exam(i.e., arterial blood pressure [BP], heart rate $[\mathrm{HR}]$, respiratory rate, oxygen saturation), height, and weight. Body mass index (BMI) is calculated from the height and weight and is more accurate than weight in establishing obesity , ASA physical status, and documentation of airway assessment, documentation of informed consent and Appropriate premedication and prophylactic antibiotic administrations.

Intraoperative anesthetic record recommendations of Committee on

Quality Management and Departmental Administration (QMDA) 
Approved by The American Society of Anesthesiologists are patient reevaluation, confirmation of necessary equipment, physiologic monitoring data, medications administered, intravenous fluids, technique(s) used and patient positioning.

Postanesthesia documentation includes patient status at transfer, significant or unexpected events/complications and presence/absence of anesthesia related complications or complaints.

\section{References}

1. Morgan \& Mikhail's Clinical Anesthesiology, 5e chapter 18.

2. Cleveland Clinic journal ofmedicine 2004.

3. Oxford Textbook of Surgery4th Edition 2004.

4. MerchantR, Bosenberg C, Brown K, Chartrand D, Dain S, Dobson J, et al. Guidelines on the practice of anaesthesia Revised 2010. Can J Anaesth. 2010;57:58-87.

5. PolanczykCA, Marcantonio E, Goldman L, et al. Impact of age on perioperative complications and length of stay in patients undergoing noncardiac surgery. Ann Intern Med; 134:637-643 2001.

6. SelzmanCH, Miller SA, Zimmerman MA, et al. The case for betaadrenergic blockade as prophylaxis against perioperative cardiovascular morbidity and mortality. Arch Surg; 136:286-290 2001 .

7. Aykut,K., Albayrak, G., Guzeloglu, M., Baysak, A., \& Hazan, E.. Preoperative mild cognitive dysfunction predicts pulmonary complications after coronary artery bypass graft surgery. Journal of Cardiothoracic and Vascular Anesthesia,1267-1270.Retrieved March12,2015,fromhttp://www.science direct.com.une.idm.oclc.org/science/art icle /pii/S105307701 3000360\#.(2013)
8. Coulter A, Collins A. Making shared decision-making a reality: no decision about me, without me. The King's Fund, London (http://bit.ly/1Qx7cQa) 2011.

9. Fleisher LA, Beckman JA, Brown KA, et al: ACCF/AHA focused update on perioperative beta blockade incorporated into the ACC/ AHA 2007 guidelines on perioperative cardiovascular evaluation and care for noncardiac surgery: a report of the American College of Cardiology Foundation/American Heart Association Task Force on Practice Guidelines, Circulation, 120:e169e276, 2009.

10. Wijeysundera DN, Austin PC, Beattie WS, et al: Variation in the practice of preoperative medical consultation for major elective noncardiac surgery: a population-based study, Anesthesiology, 116:25-34, 2012.

11. Sharma G, Kuo YF, Freeman J, et al: Comanagement of hospitalized surgical patients by medicine physicians in the United States, Arch Intern, , Med 170:363-368 2010 .

12. Goldman, L., Caldera, D., Nussbaum, S., Southwick, F., Krogstad, D., Murray, B., Slater, E. (1977). Multifactorial index of cardiac risk in noncardiac surgical procedures. New England Journal of Medicine, 297(16), 845-850. Retrieved February 28, from http://www.nejm.org.une.idm.oclc.org/ doi/full/10.1056/NEJM1977102029716 0,2015 .

13. Lee, T., Marcantonio, E., Mangione, C., Thomas, E., Polanczyk, C., Cook, E., . . G Goldman, L. (1999). Derivation and prospective validation of a simple index for prediction of cardiac risk of major noncardiac surgery. Circulation, 100(10), 1043-1049. Retrieved February 28, , 2015

14. Mazo, V., Sabaté, S., Canet, J., Gallart, L., Gama de Abreu, M., Belda, 
J., Pelosi, P. Prospective external validation of a predictive score for postoperative pulmonary complications. Anesthesiology, (2014).V 121(2), 219-231. Retrieved January 13, 2015.

15. Kim, S., Brooks, A., Groban, L. (2015). Preoperative assessment of the older surgical patient: Honing in on geriatric syndromes. Clinical Interventions in Aging, 10, 13-27. Retrieved February 17, from http://dx.doi.org.une.idm.oclc.org/10.2 147/CIA.S75285, 2015.

16. Hulzebos EH, Helders PJ, Favie NJ, De Bie RA, Brutel de la Riviere A, Van Meeteren NL. Preoperative intensive inspiratory muscle training to prevent postoperative pulmonary complications in high-risk patients undergoing $\mathrm{CABG}$ surgery: a randomized clinical trial. JAMA. Oct 18. 296(15):1851-7 2006 .

17. Wu CC, Yeh DC, Lin MC, Liu TJ, P'Eng FK. Improving operative safety for cirrhotic liver resection. Br J Surg. $2001 \mathrm{Feb} ; 88(2): 210-5$.

18. Befeler AS, Palmer DE, Hoffman M, et al: The safety of intra-abdominal surgery in patients with cirrhosis: model for end-stage liver disease score is superior to Child-Turcotte-Pugh classification in predicting outcome, Arch Surg 140:650-654, , discussion, $p$ 655. 2005

19. Pearse RM, Moreno RP, Bauer $P$, et al: Mortality after surgery in Europe: a 7 day cohort study, Lancet, 380:10591065, 2012.

20. Rosenman DJ, McDonald FS, Ebbert $\mathrm{JO}$, et al: Clinical consequences of withholding versus administering renin-angiotensinaldosterone system antagonists in the preoperative period, J Hosp Med, 3:319-325, 2008.

21. Kheterpal S, Khodaparast O, Shanks A, et al: Chronic angiotensinconverting enzyme inhibitor or angiotensin receptor blocker therapy combined with diuretic therapy is associated with increased episodes of hypotension in noncardiac surgery, $\mathbf{J}$ Cardiothorac Vasc Anesth, 22:180-186, 2008.

22. Khan NA, Campbell NR, Frost SD, et al: Risk of intraoperative hypotension with loop diuretics: a randomized controlled trial, Am J Med, 123:1059.e1-e8, 2010 .

23. Horlocker TT, Wedel DJ, Rowlingson JC, et al: Regional anesthesia in the patient receiving antithrombotic or thrombolytic therapy: American Society of Regional Anesthesia and Pain Medicine evidence- based guidelines (third edition), Reg Anesth Pain Med, 35: 64101, 2010.

24. Grady D, Wenger NK, Herrington D, et al: Postmenopausal hormone therapy increases risk for venous thromboembolic disease: the Heart and Estrogen/progestin Replacement Study, Ann Intern Med, 132:689-696,2000.

25. QMDA approved by ASA 2015 .

26. Swart M, Houghton K. Pre-operative preparation: essential elements for delivering enhanced recovery pathways. Curr Anaesth Crit Care;21:142-147 2010.

27. Mutcherson KM: Whose body is it anyway? An updated model of healthcare decision-making rights for adolescents, Cornell J Law Public Policy, 2005, 14(25):251-325.

28. SimpsonJC et al. Enhanced recovery from surgery in the UK: an audit of the enhanced recovery partnership programme 2009-2012. $\mathrm{Br}$ J Anaesth;115:560-568 2015 .

29. Davies JM: Consent in laboring patients. In Van Norman G, Jackson S, Rosenbaum S, Palmer S, editors: Clinical ethics in anesthesiology: a case-based textbook. Cambridge, , Cambridge University Press, pp 44-48, 2011..

30. McCombe K, Bogod DG. Paternalism and consent: has the law 
SOHAG MEDICAL JOURNAL

Vol. 21 No.3 october 2017

finally caught up with the profession? Anaesthesia;70:1016-1019 2015.

31. azirani S, Lankarani-Fard A, Liang L-J, et al: Perioperative processes and outcomes after implementation of a
Recent guidelines in preoperative assessment Sara M.Galal. Et al

hospitalist-run preoperative clinic, $\mathrm{J}$ Hosp Med, 7:697-701, 2012.

32. Public Hospitals Act, Revised Statutes of Ontario, 1990.

33. $\quad$ QMDA approved by ASA 2015. 